\title{
TITLE:
}

\section{ON BLOCH FUNCTIONS AND THE CONTRACTION OF TEICHMULLER METRICS}

$\operatorname{AUTHOR}(\mathrm{S})$ :

XINZHONG, HUANG; OWA, SHIGEYOSHI

\section{CITATION:}

XINZHONG, HUANG ...[et al]. ON BLOCH FUNCTIONS AND THE CONTRACTION OF TEICHMULLER METRICS. 数理解析研究所講究録 1997, 1012: 198-204

ISSUE DATE:

1997-09

URL:

http://hdl.handle.net/2433/61533

RIGHT: 


\title{
ON BLOCH FUNCTIONS AND THE CONTRACTION OF TEICHMÜLLER METRICS
}

\author{
HuANg Xinzhong AND Shigeyoshi OWA
}

\begin{abstract}
In this note, we consider the properties of Bloch functions determined by Beltrami coefficient. A sufficient condition for extremal quasiconformal mapping with nonexistence of degenerating sequence is obtained. As a result, we consider the contraction or preserved of Teichmüller metrics for the related Beltrami lines under the projection mapping $\pi$.
\end{abstract}

\section{INTRODUCTION}

Let $Q_{I}$ be the class of quasiconformal mappings $f$ of the unit disk $D=\{z \| z \mid \leq 1\}$ onto itself with $f(0)=f(1)-1=0, \mu_{f}$ be the complex dilatation of $f, k_{f}=$ $\left\|\mu_{f}\right\|_{\infty}=\operatorname{esssup}_{z \in D}\left|\mu_{f}\right|, k_{0}(f)=\inf _{g} k_{g}$, where $g \in Q_{I}$ with $\left.g\right|_{\partial D}=\left.f\right|_{\partial D}$. We say that $f(z)$ is extremal if $k_{f}=k_{0}(f)$, and the corresponding $\mu_{f}$ is called extremal.

We know that the universal Teichmüller space $T(1)$ can be represented as a quotient space of $Q S$ by the Möbius group $P S L(2, R)$, where $Q S$ is the group of all quasi-symmetric homeomorphisms of a circle, and the Teichmüller distance $d([f],[g])$, from a point $[g]$ to another point $[f]$ in $T(1)$, is equal to

$$
d([f],[g])=\frac{1}{2} \log \frac{1+k_{0}\left(g \circ f^{-1}\right)}{1-k_{0}\left(g \circ f^{-1}\right)}
$$

$Q S$ contains another topological subgroup, which is much larger than $P S L(2, R)$, the subgroup $S$ of symmetric homeomorphisms. Gardiner-Sullivan [1] showed that $Q S \bmod S$ also has a natural complex Banach manifold structure and a natural quotient metric $\bar{d}$, called the Teichmüller metric in $Q S \bmod S$. Let $\bar{k}_{f}=$ $\inf _{U} \operatorname{esssup}_{z \in U}\left|\mu_{f}(z)\right|$, where $U$ moves all neighborhoods of $\partial D$ in $D, \bar{k}_{f}$ is called the boundary dilatation of $f$. Set $\bar{k}_{0}(f)=\inf _{g} \bar{k}_{g}$, where $g$ moves all quasiconformal mappings of $D$ with the same boundary values as $f$. If $\bar{k}_{0}(f)=\bar{k}_{f}$, then $f(z)$ is called extremal in $Q S \bmod S$. The distance between two points $\pi[f]$ and $\pi[g]$ in $Q S m o d S$ is equal to

$$
\bar{d}(\pi[f], \pi[g])=\frac{1}{2} \log \frac{1+\bar{k}_{0}\left(g \circ f^{-1}\right)}{1-\bar{k}_{0}\left(g \circ f^{-1}\right)} .
$$

Suppose $\mu(z)$ is a given Beltrami coefficient, we consider the Beltrami line $C_{\mu}=$ $\left\{\left[f^{t}\right] \mid-1 \leq t \leq 1\right\}$ or $\pi C_{\mu}=\left\{\pi\left[f^{t}\right] \mid-1 \leq t \leq 1\right\}$, where $\mu_{f^{t}}=t \frac{\mu}{\|\mu\|_{\infty}}$. If $\mu$ is

The first author was supported by the National Science Foundation of Fujian, China . 
extremal in $T(1)$ or in $Q S \bmod S$, then the natural mapping $t \mapsto t \frac{\mu}{\|\mu\|_{\infty}}$ from the open interval $(-1,1)$ with the Poincaré metric onto $C_{\mu}$ or $\pi C_{\mu}$ with the Teichmüller metric is an isometry. Whether $\mu$ is extremal or not, such mapping is weakly contracting. The following problem is very interesting and considered by many authors(cf. [2],[3]):

For which points $[f] \in T(1)$, does the Teichmuller distance from 0 to $[f]$ in $Q S$ strictly greater than the distance from 0 to $\pi[f]$ in $Q S \bmod S$ ?

In this note, we will investigate some properties for Bloch functions determined by $\mu$ and partially solve the above problem.

\section{MAIN RESULTS AND THEIR PROOFS}

Let $f(z)=\sum_{n=0}^{\infty} a_{n} z^{n}$ be analytic in $D, f(z)$ is called a Bloch function if

$$
\|f\|_{B}=\sup _{z \in D}\left(1-|z|^{2}\right)\left|f^{\prime}(z)\right|<\infty
$$

The Bloch functions will be denoted by $B . B_{0}$ will be the subset of $B$ with

$$
\|f\|_{B_{0}}=\lim _{|z| \rightarrow 1} \sup \left(1-|z|^{2}\right)\left|f^{\prime}(z)\right|=0
$$

$A(D)=\left\{f(z) \mid \mathrm{f}(\mathrm{z})\right.$ is analytic in $\left.\mathrm{D},\|f(z)\|_{1}=\frac{1}{\pi} \iint_{D}|f(z)| d x d y<\infty\right\}$. The quasiconformal mapping $f$ from $D$ onto itself is called a Teichmüller mapping of finite type, if $\mu_{f}=\|\mu(z)\|_{\infty} \frac{\bar{\varphi}_{0}}{\varphi_{0} \mid}, \varphi_{0} \in A(D)$. From Reich's example(cf.[4]), we know that even the point $[f]$ corresponds to a Teichmüller mapping of finite type, the distance from 0 to $[f]$ under the projection $\pi$ may not contract. However, if $[f] \in T(1)$, and $\bar{d}(0, \pi[f])<d(0,[f])$, then $[f]$ contains a Teichmüller mapping of finite type. This makes the above problem more complicated.

Suppose $\kappa(z) \in L^{\infty}(D)$, the space of complex-valued bounded measurable functions in $D$ with $\|\kappa\|_{\infty}=\operatorname{esssup}_{z \in D}|\kappa(z)|$, we consider a linear functional $L_{\kappa}$ on $A(D)$

$$
L_{\kappa}(f)=\frac{1}{\pi} \iint_{D} \kappa(z) f(z) d x d y, \quad f(z) \in A(D)
$$

then

$$
\left\|L_{\kappa}\right\| \leq\|\kappa\|_{\infty}
$$

Hamilton, Reich and Streble [5, 6] showed that

Theorem A. A Beltrami coefficient $\mu$ is extremal if and only if one of the following statements holds:

1) There exist $\varphi \in A(D)$ and $k \in[0,1)$ such that $\mu=k \bar{\varphi} /|\varphi|$ for almost everywhere on $D$.

2) There is a degeneration sequence $\left\{\varphi_{n}\right\} \in A(D),\left\|\varphi_{n}\right\|_{1}=1$, converging to 0 locally uniformly in $D$, such that 


$$
\lim _{n \rightarrow \infty}\left|\iint_{D} \varphi_{n} \mu d x d y\right|=\|\mu\|_{\infty}
$$

For a given Beltrami coefficient $\mu(z)$, let

$$
b_{n}=\frac{n+2}{\pi} \iint_{D} \mu(z) z^{n} d x d y, \quad g(\zeta)=\sum_{n=0}^{\infty} b_{n} \zeta^{n}
$$

it is clearly that $\left|b_{n}\right| \leq 2\|\mu(z)\|_{\infty}$ and $g(\zeta)$ is analytic in $D$. We call that the analytic function $g(\zeta)$ is determined by $\mu(z)$.

Let $G(\zeta)=\zeta g(\zeta)$, Anderson proved in [7] the following

Theorem B. For a given $\mu(z) \in L^{\infty}(D)$, then

$$
\left\|L_{\mu}\right\| \leq\|G(\zeta)\|_{B} \leq 4\left\|L_{\mu}\right\|
$$

where $G^{\prime}(\zeta)=\frac{2}{\pi} \iint_{D} \frac{\mu(z)}{(1-\zeta z)^{3}} d x d y$.

Theorem C. If $\mu(z)$ possesses a degenerating sequence, then

$$
\left\|L_{\mu}\right\| \leq \lim _{|z| \rightarrow 1} \sup \left(1-|z|^{2}\right)\left|G^{\prime}(z)\right|
$$

where $G^{\prime}(\zeta)=\frac{2}{\pi} \iint_{D} \frac{\mu(z)}{(1-\zeta z)^{3}} d x d y$. In particular, if

$$
\iint_{D} \frac{\mu(z)}{(1-\zeta z)^{3}} d x d y=o\left(1-|\zeta|^{2}\right)^{-1} \quad\left(|\zeta| \rightarrow 1^{-}\right),
$$

then $\mu(z)=\|\mu\|_{\infty} \frac{\bar{\varphi}_{0}(z)}{\varphi_{0}(z)}, \varphi_{0} \in A(D)$, for almost all $z \in D$.

Theorem $C$ means that if $\mu(z)$ is extremal and $\lim _{|z| \rightarrow 1} \sup \left(1-|z|^{2}\right)\left|G^{\prime}(z)\right|=0$, then

$$
\mu(z)=\|\mu\|_{\infty} \bar{\varphi}_{0} /\left|\varphi_{0}\right|, \quad \varphi_{0}(z) \in A(D)
$$

for almost everywhere $z \in D$. For an extremal quasiconformal mapping $f^{\mu(z)} \in Q_{I}$, in what case, is it a finite type Teichmüller mapping or even has it no degenerating sequence? This problem is very interesting itself(cf. $[8,9]$ and the references cited there). First, we will prove the following

Theorem 1. Suppose $\mu(z)$ is extremal, let $g(z)$ be defined in (2.6), if there exists a $\rho_{0}, 0<\rho_{0}<1$, such that

$$
\sup _{\rho_{0}<|z|<1}\left(1-|z|^{2}\right)\left|g^{\prime}(z)\right|<1
$$

then there exists a $\varphi_{0} \in A(D)$ with $\mu(z)=\|\mu(z)\|_{\infty} \frac{\bar{\varphi}_{0}}{\left|\varphi_{0}\right|}$ for almost all $z \in D$. In particular, $\mu(z)$ possesses no degenerating sequence.

The proof of Theorem 1. If $\mu(z)$ is an extremal Beltrami coefficient, let $g(\zeta)$ be defined in (2.6), if $f(z)=\sum_{n=0}^{\infty} a_{n} z^{n} \in A(D), 0<\rho<1$, we have

$$
L_{\mu}(f(\rho z))=\sum_{n=0}^{\infty} a_{n} \rho^{n} L_{\mu}\left(z^{n}\right)=\sum_{n=0}^{\infty} \frac{a_{n} b_{n}}{n+2} \rho^{n} .
$$


Since $\|f(\rho z)-f(z)\|_{1} \rightarrow 0$, when $\rho \rightarrow 1^{-}$, then we have

$$
L_{\mu}(f)=\lim _{\rho \rightarrow 1^{-}} \sum_{n=0}^{\infty} \frac{a_{n} b_{n}}{n+2} \rho^{n} .
$$

On the other hand, if $G(\zeta)=\zeta g(\zeta)$, then

$$
\begin{aligned}
& \frac{1}{2 \pi} \int_{0}^{2 \pi} f\left(r e^{i \theta}\right) G^{\prime}\left(\zeta r e^{-i \theta}\right) d \theta \\
& =\frac{1}{2 \pi} \int_{0}^{2 \pi}\left(\sum_{n=0}^{\infty} a_{n} r^{n} e^{i n \theta}\right)\left(\sum_{n=0}^{\infty}(n+1) b_{n} \zeta^{n} r^{n} e^{-i n \theta}\right) d \theta \\
& =\sum_{n=0}^{\infty}(n+1) a_{n} b_{n} \zeta^{n} r^{2 n} .
\end{aligned}
$$

Thus, we have

$$
\lim _{\rho \rightarrow 1^{-}} \sum_{n=0}^{\infty} \frac{a_{n} b_{n}}{n+2} \rho^{n}=\frac{1}{\pi} \int_{0}^{1} \int_{0}^{2 \pi} f\left(r e^{i \theta}\right) G^{\prime}\left(\zeta r e^{-i \theta}\right)\left(1-r^{2}\right) r, d r d \theta
$$

for any $f(z) \in A(D)$. Since

$$
\begin{aligned}
g(\zeta)=\sum_{n=0}^{\infty} b_{n} \zeta^{n} & =\sum_{n=0}^{\infty}\left(\frac{n+2}{\pi} \iint_{D} z^{n} \mu(z) d x d y\right) \zeta^{n} \\
& =\frac{1}{\pi} \iint_{D}\left(\sum_{n=0}^{\infty}(n+2) z^{n} \zeta^{n} \mu(z)\right) d x d y \\
& =\frac{1}{\pi} \iint_{D}\left[\frac{2-z \zeta}{(1-z \zeta)^{2}}\right] \mu(z) d x d y
\end{aligned}
$$

then,

$$
|g(\zeta)| \leq \frac{3\|\mu\|_{\infty}}{\pi|\zeta|} \log \frac{1+|\zeta|}{1-|\zeta|}=o\left(\left(1-|\zeta|^{2}\right)^{-1}\right), \quad|\zeta| \rightarrow 1^{-} .
$$

If $\left\{f_{n}(z)\right\}$ is a degenerating sequence for $\mu(z)$ with $\left\|f_{n}\right\|_{1}=1$, by Theorem $\mathrm{B}$ and (2.11), we can choose a $\rho^{\prime}$ with $\rho_{0}<\rho^{\prime}<1$ such that

$$
\begin{aligned}
\left|L_{\mu}\left(f_{n}\right)\right| & \leq \frac{4\|\mu\|_{\infty}}{\pi} \iint_{|z| \leq \rho^{\prime}} \mid f_{n}\left(r e^{i \theta)}\left|r d r d \theta+\sup _{\rho^{\prime}<|z|<1}\left(1-|z|^{2}\right)\right| g(z) \mid\right. \\
& +\sup _{\rho^{\prime}<|z|<1}\left(1-|z|^{2}\right)\left|g^{\prime}(z)\right|<1, \quad \text { for } n \rightarrow \infty,
\end{aligned}
$$

which contradicts that $\left\{f_{n}(z)\right\}$ is a degenerating sequence. By Theorem $\mathbf{A}$, Theorem 1 is proved.

The following example 1 shows that there is non-extremal Beltrami coefficient $\mu(z)$ with the bound $\sup _{p_{0}<|z|<1}\left(1-|z|^{2}\right)\left|g^{\prime}(z)\right|=\frac{2}{\pi}$. 
Example 1. Set Beltrami coefficient

$$
\mu(z)= \begin{cases}1, & \text { for } \Im z \geq 0,|z|<1 \\ 0, & \text { for } \Im z<0,|z|<1\end{cases}
$$

Then by $[8$, Theorem 1$]$, we see that $\mu(z)$ is not extremal. In this case, by calculation, we have

$$
g^{\prime}(z)=2+\frac{2 i}{\pi}\left[z+\frac{1}{3} z^{3}+\cdots+\frac{1}{2 n-1} z^{2 n-1}+\cdots\right]
$$

and $\lim _{|z| \rightarrow 1}\left(1-|z|^{2}\right)\left|g^{\prime}(z)\right|=\frac{2}{\pi}$.

Next we will investigate the relationship between extremal Beltrami coefficient $\mu$ and the coefficients of $g(z)$ defined in (2.6).

From [11] and Theorem 1, we know that if $\mu(z)$ is extremal and the determained analytic function $g(z) \in B_{0}$, then $\lim _{n \rightarrow \infty}\left|b_{n}\right|=0$. However, we also know that even if $f(z) \in B$ and $\lim _{n \rightarrow \infty}\left|b_{n}\right|=0$, one can not derive that $f(z) \in B_{0}$. From this we will prove the following

Corollary 1. Suppose $\mu(z)$ is extremal, and let $g(z)=\sum_{n=0}^{\infty} b_{n} z^{n}$ be defined in (2.6), if there exist a positive number $N_{0}$ and $l, 0<l<\frac{1}{2}$, such that

$$
\left|b_{n}\right|<\frac{l}{n}, \quad \text { holds for } n>N_{0},
$$

then there exists a $\varphi_{0}(z) \in A(D)$ with

$$
\mu(z)=\|\mu\|_{\infty} \bar{\varphi}_{0} /\left|\varphi_{0}\right|, \quad \text { for almost all } z \in D \text {. }
$$

The proof of Collary 1. If $\mu(z)$ is extremal, and let $g(z)=\sum_{n=0}^{\infty} b_{n} z^{n}$ be defined in (2.6), we have

$$
\begin{aligned}
\left|g^{\prime}(z)\right| & \leq\left|\sum_{n=0}^{N_{0}} n b_{n} z^{n}\right|+\sum_{n=N_{0}+1}^{\infty} l\left|z^{n}\right| \\
& =\left|\sum_{n=0}^{N_{0}} n b_{n} z^{n}\right|+l \frac{|z|^{N_{0}+1}}{1-|z|}
\end{aligned}
$$

thus there exists a $\rho_{0}>0$, such that $\sup _{\rho_{0}<|z|<1}\left(1-|z|^{2}\right)\left|g^{\prime}(z)\right|<1$, by Theorem 1 , we obtain the assertion.

Let $\Pi$ denote the subset of $T(1)$ consisting of elements of $[f]$ which correspond to Teichmüller mappings of finite type whose complex dilatations $\mu=\mu_{f}$ satisfy the following condition: There exists a $\rho_{0}, 0<\rho_{0}<1$, such that $\sup _{\rho_{0}<|\zeta|<1}(1-$ $\left.|\zeta|^{2}\right)\left|g^{\prime}(\zeta)\right|<1$, where $g(\zeta)$ is defined in (2.6). We will prove the following

Theorem 2. For $[f] \in \Pi$, then $\bar{d}(0, \pi([f]))<d(0,[f])$. [2].

In order to prove Theorem 2, we need the following Theorem D due to Gardiner 
Theorem D. For every $[f] \in T(1)$, then $\bar{k}_{f}=\bar{k}_{0}(f)$ if and only if

$$
\sup _{\left\{\varphi_{n}\right\}} \limsup _{n \rightarrow \infty}\left|\operatorname{Re} \iint_{D} \varphi_{n} \mu_{f} d x d y\right|=\bar{k}_{f}
$$

where the supremum is taken over all degenerating sequences $\left\{\varphi_{n}\right\}$ for $\mu_{f}$ with $\left\|\varphi_{n}\right\|_{1}=1$ in $A(D)$.

The proof of Theorem 2. We use the same way as in [3] to prove Theorem 2. If $[f] \in \Pi$, then we conclude that $\bar{k}_{0}(f)=k_{0}(f)$. On the contrary, by Theorem $\mathrm{D}$, we can find a degenerating sequence $\left\{\varphi_{n}\right\}$ with $\left\|\varphi_{n}\right\|_{1}=1$ such that

$$
\lim _{n \rightarrow \infty} \operatorname{Re} \iint_{D} \varphi_{n} \mu_{f} d x d y=\left\|\mu_{f}\right\|_{\infty}=k_{0}(f)=\bar{k}_{0}(f)
$$

which is impossible by Theorem 1 .

Thus we have $\bar{k}_{0}(f)<k_{0}(f)$, which is equivalent to $\bar{d}(0, \pi([f]))<d(0,[f])$.

On the other hand, comparing with Theorem 2, we will prove the following

Theorem 3. Suppose $[f] \in T(1)$, and $b_{n}=\frac{n+2}{\pi} \iint_{D} \mu_{f} z^{n} d x d y$, if $\varlimsup_{n \rightarrow \infty} b_{n}=$ $2\left\|\mu_{f}\right\|_{\infty}$, then $\bar{d}(0, \pi([f]))=d(0,[f])$. The constant 2 is the best.

The proof of Theorem 3. First, from Fehlmann and Sakan's paper in [10], we know that the subset of $T(1)$ satisfying the conditions in Theorem 3 is not empty, and by the example of Fehlmann and Sakan made in [10], there exists an extremal Beltrami coefficient $\mu$ such that the coefficients of $g(z)$ satisfy $\overline{\lim }_{n \rightarrow \infty} b_{n}=2\|\mu\|_{\infty}$, thus the constant 2 is the best. Now, if $\overline{\lim }_{n \rightarrow \infty} b_{n}=2\left\|\mu_{f}\right\|_{\infty}$, then we have $\lim _{j \rightarrow \infty} b_{n_{j}}=2\left\|\mu_{f}\right\|_{\infty}$, and the sequence $\left\{\varphi_{n_{j}}(z)=\frac{n_{j}+2}{2} z^{n_{j}}\right\}$ is a degenerating sequence for the Beltrami coefficient $\mu_{f}$, with $\left\|\varphi_{n_{j}}\right\|_{1}=1$, by Theorem $\mathrm{D}$, we conclude that $\bar{k}_{0}(f)=k_{0}(f)$, thus $d(0, \pi([f]))=d(0,[f])$.

To consider the contraction of Teichmüller metrics, we need the following Principle of Teichmüller contraction due to Gardiner [2].

Principle of Teichmüller contraction. Assume $\|\mu\|=1,0<k_{1}<k_{2}<1$, and $d\left(0,\left[f^{k_{1}}\right]\right) \leq \lambda_{1} d_{p}\left(0, k_{1}\right)$ or $\bar{d}\left(0, \pi\left(\left[f^{k_{1}}\right]\right)\right) \leq \lambda_{1} d_{p}\left(0, k_{1}\right)$ with some $\lambda_{1}<1$, where and in the sequel, $f^{k}$ is the quasiconformal mapping of $D$ on to itself such that $\mu_{f}=k \mu$ for every positive $k<1$. Then there exists a $\lambda_{2}<1$ depending only on $k_{1}, k_{2}$, and $\lambda_{1}$ such that

$$
d\left(0,\left[f^{k}\right]\right) \leq \lambda_{2} d_{p}(0, k) \quad \text { or } \quad \bar{d}\left(0, \pi\left(\left[f^{k}\right]\right)\right) \leq \lambda_{2} d_{p}(0, k)
$$

respectively, for all $k$ with $0 \leq k \leq k_{2}$.

Using Theorem 2 and the Principle of Teichmüller contraction, we can obtain the following 
Corollary 2. Under the same circumstance as in Theorem 2 , let $k=\left\|\mu_{f}\right\|_{\infty}$ and $\lambda=\bar{d}(0, \pi([f])) / d(0,[f])$. Fix $k^{\prime}<1$ and let $f^{t}$ be the quasiconformal mapping of $D$ onto itself such that $\mu_{f^{t}}=(t / k) \mu_{f}$ for every $t \in\left[0, k^{\prime}\right)$. Then there exists $\lambda^{\prime}<1$ depending only on $k, k^{\prime}$, and $\lambda$ such that

$$
\bar{d}\left(0, \pi\left(\left[f^{t}\right]\right)\right) \leq \lambda^{\prime} d_{p}(0, t)
$$

for every $t$ with $0 \leq t \leq k^{\prime}$, where $d_{p}$ denotes the Poincaré metric on $D$.

The proof of Corollary 2. By Theorem 2, we have $d(0,[f])=d_{p}(0, k)$ and $\lambda=$ $\bar{d}(0, \pi[f]) / d(0,[f])<1$, using the principle of Teichmüller contraction, the Corollary 2 is obtained.

\section{REFERENCES}

1. F. P. Gardiner and D. Sullivan, Symmetric and quasisymmetric structures on a closed curve, Amer: J. Math. 114 (1992), 683-736.

2. F. P. Gardiner, On Teichmüller contraction, Proc. Amer. Math. Soc. 118 (1993), 865-875.

3. X. Z. Huang and M. Tanignchi, On the contraction of Teichmüller metrics, J. Math. Kyoto Univ. 35 (1995), 133-142.

4. E. Reich, An extremum problem for analytic functions with area norm, Ann. Acad. Sci. Fenn. 2 (1976), 429-445.

5. R. S. Hamilton, Extremal quasiconformal mappings with given boundary values, Trans. Amer. Math. Soc. 138 (1969), 399-406.

6. E. Reich and K. Strebel, Extremal quasiconformal mappings with given boundary values, Contributions to Analysis, Academic Press (1974), 375-391.

7. J. M. Anderson, The extremum problem for analytic functions with finite area integral, Comment. Math. Helv. 55 (1980), 87-96.

8. X. Z. Huang, The image domain of an extremal dilatation, Advances in Math. (China) 22 (1993), 435-446.

9. X. Z. Haang, On the extremality for Teichmüller mappings, J. Math. Kyoto Univ. 35 (1995), 115-132.

10. R. Fehlmann and K. Sakan, On the set of substantial boundary points for extremal quasiconformal mappings, Complex Variables 6 (1986), 323-335.

11. J. M. Anderson, J. Clunie and Ch. Pommerenke, On Bloch functions and normal functions, J. Reine Angew. Math. 270 (1974), 12-37.

Department of Mathematics, Huaqiao Univerity, Quanzhou, Fujian 362011, China Department of Mathematics, Kinki Univerity, Higashi-Osaka, Osaka 577, Japan 